\title{
Large-scale pharmacogenomics based drug discovery for ITGB3 dependent chemoresistance in mesenchymal lung cancer
}

\author{
Soon-Ki Hong ${ }^{1 \dagger}$, Haeseung Lee ${ }^{2+}$, Ok-Seon Kwon ${ }^{1 \dagger}$, Na-Young Song ${ }^{1}$, Hyo-Ju Lee ${ }^{3}$, Seungmin Kang ${ }^{2}$, \\ Jeong-Hwan $\mathrm{Kim}^{4}$, Mirang Kim${ }^{4}$, Wankyu Kim ${ }^{2^{*}}$ and Hyuk-Jin Cha ${ }^{1 *}$ (1)
}

\begin{abstract}
Even when targets responsible for chemoresistance are identified, drug development is often hampered due to the poor druggability of these proteins. We systematically analyzed therapy-resistance with a large-scale cancer cell transcriptome and drug-response datasets and predicted the candidate drugs based on the gene expression profile. Our results implicated the epithelial-mesenchymal transition as a common mechanism underlying resistance to chemotherapeutic drugs. Notably, we identified ITGB3, whose expression was abundant in both drug resistance and mesenchymal status, as a promising target to overcome chemoresistance. We also confirmed that depletion of ITGB3 sensitized cancer cells to conventional chemotherapeutic drugs by modulating the NF-KB signaling pathway. Considering the poor druggability of ITGB3 and the lack of feasible drugs to directly inhibit this protein, we took an in silico screening for drugs mimicking the transcriptome-level changes caused by knockdown of ITGB3. This approach successfully identified atorvastatin as a novel candidate for drug repurposing, paving an alternative path to drug screening that is applicable to undruggable targets.
\end{abstract}

Keywords: Chemoresistance, Mesenchymal cancer, Pharmacogenomics, Drug repurposing, Biomarker, ITGB3, NF-KB, Atorvastatin, Systems pharmacology

\section{Main text}

Recent studies in both in vitro cell and in vivo animal models demonstrated that the epithelial-mesenchymal transition (EMT), a major cause of metastasis, is closely associated with chemoresistance [1]. These are consistent with the reports that cancer patients with mesenchymal gene signatures have poor prognoses or exhibit therapy resistance [2]. However, due to the poor druggability of the EMT-associated proteins responsible for chemoresistance (e.g., ZEB1/2, SNAI2, SOX4, etc.), it is important to develop alternative strategies to make 'undruggable but attractive targets' druggable.

\footnotetext{
* Correspondence: wkim@ewha.ac.kr; hjcha93@snu.ac.kr

${ }^{\dagger}$ Soon-Ki Hong, Haeseung Lee and OK-Seon Kwon contributed equally to this work.

2Department of Life Sciences, Ewha Womans University, Seoul 03760,

Republic of Korea

${ }^{1}$ College of Pharmacy, Seoul National University, Seoul 08826, Republic of Korea

Full list of author information is available at the end of the article
}

To elucidate the mechanisms underlying chemoresistance, we examined the gene expression profiles of 804 cancer cell lines, as well as their responses to anti-cancer drugs, using data from Cancer Therapeutics Response Portal (CTRP) (Additional file 1: Figure $\mathrm{S} 1 \mathrm{~A})$. For each drug, cell lines were classified as resistant or sensitive group, and differentially expressed genes (DEGs) in each resistant group were selected (Additional file 1: Figure S1B). EMT was the most frequently up-regulated phathway in the resistant group across most chemotherapeutics (26 out of 32 drugs), and targeted drugs (15 out of 20 drugs) (Fig. 1a and Additional file 1: Figure S1C). Among the downregulated genes, 'immune \& inflammatory response' pathways were highly enriched (Additional file 1: Figure S1C). It would be noteworthy that upregulation of interferon signaling contributes to efficacy of chemotherapy [3]. Given the previous studies supporting EMT as

(C) The Author(s). 2018 Open Access This article is distributed under the terms of the Creative Commons Attribution 4.0 International License (http://creativecommons.org/licenses/by/4.0/), which permits unrestricted use, distribution, and 
A

$$
\begin{gathered}
\text { Epithelial mesenchymal transition } \\
\text { Angiogenesis } \\
\text { Coagulation } \\
\text { KRAS signaling dn } \\
\text { KRAS signaling up } \\
\text { Myogenesis } \\
\text { Hypoxia } \\
\text { Inflammatory response } \\
\text { TNFa signaling via NF-kB } \\
\text { Complement } \\
\text { Estrogen response early } \\
\text { Estrogen response late } \\
\text { Apical junction } \\
\text { Xenobiotic metabolism } \\
\text { UV response dn } \\
\qquad \begin{array}{ccc}
\hline \\
\text { Chemotherapeutic drugs }
\end{array}
\end{gathered}
$$

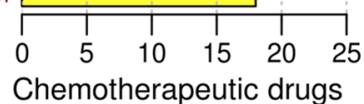

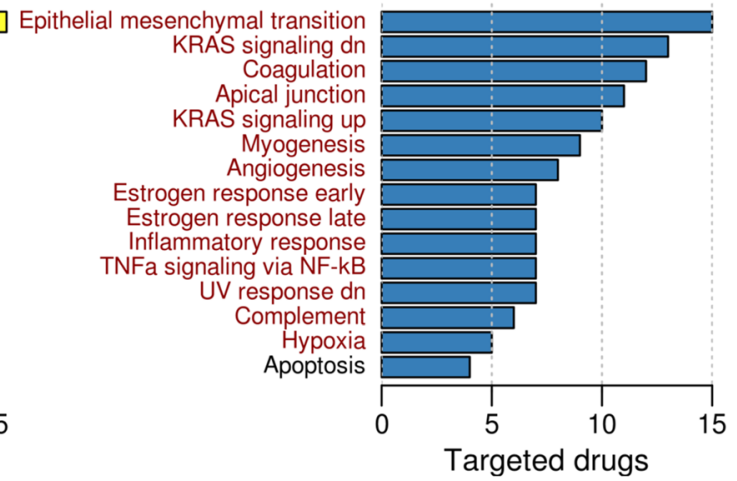

B

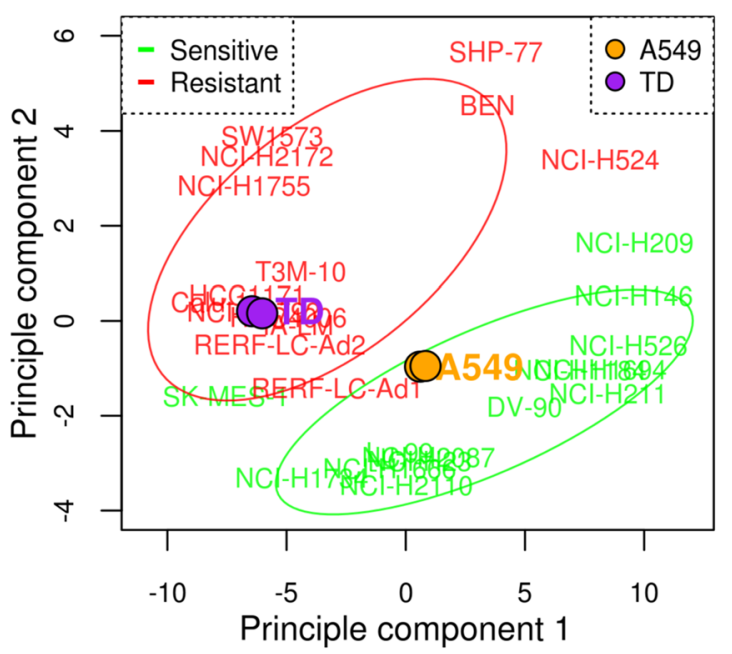

D

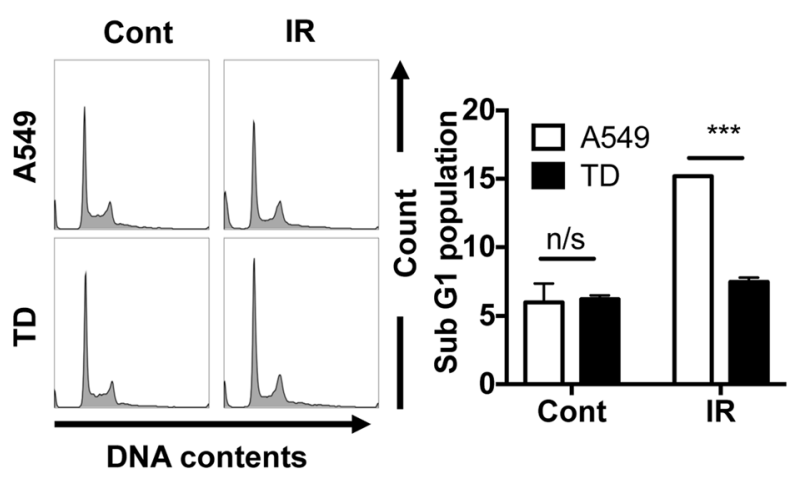

C

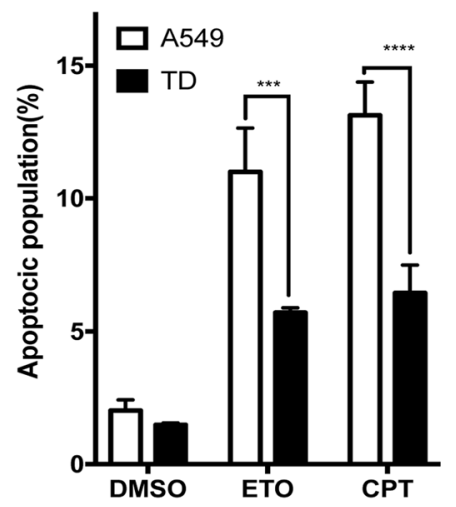

E

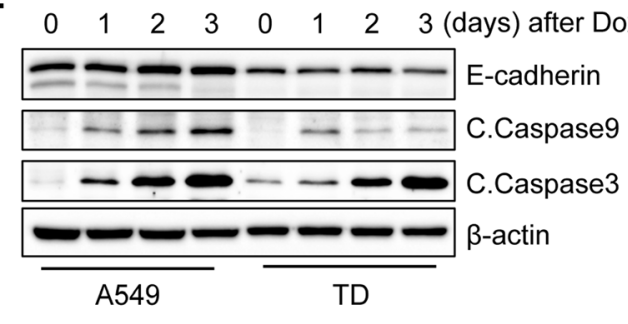

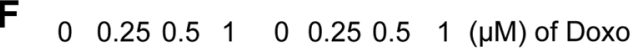

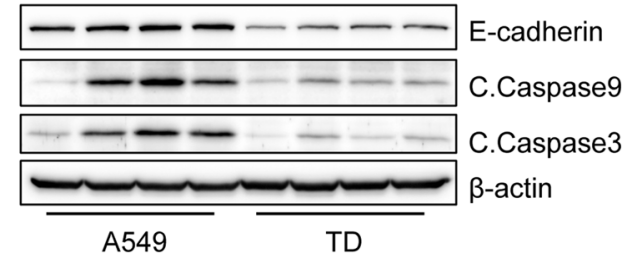

Fig. 1 Epithelial mesenchymal transition as a common mechanism underlying anticancer drug resistance a Top 10 most up-regulated pathways in the resistance group across chemotherapeutic (left panel) and targeted drugs (right panel) are summarized as the number of drugs by which the corresponding pathway is significantly regulated. Significantly enriched pathways per a drug were selected through hypergeometric tests (FDR < 0.05) using the hallmark gene sets from MsigDB. $\mathbf{b}$ Clustering of A549 and TD cells together with other lung cancer cell lines from the resistant (red) and sensitive (green) groups for doxorubicin by Partial Least Square Discriminant Analysis (PLS-DA) based on known EMTgenes c Programed cell death was examined by Annexin V/7AAD staining after DMSO, etoposide (ETO: $80 \mu \mathrm{M}$ ) and Camptothecin (CPT: $1 \mu$ M) $48 \mathrm{~h}$ treatment. $\mathbf{d}$ Sub G1 population was measure by FACS at $48 \mathrm{~h}$ after IR. The quantified sub G1 population was presented as bar graph (right) e and $\mathbf{f}$ Immunoblotting for apoptosis marker such as cleaved caspase 3 and 9 (C.Caspase3 and 9) after doxorubicin (Doxo) treatment at indicative days (e) or concentration (f), $\beta$-actin and E-cadherin used for an equal loading control and epithelial marker 


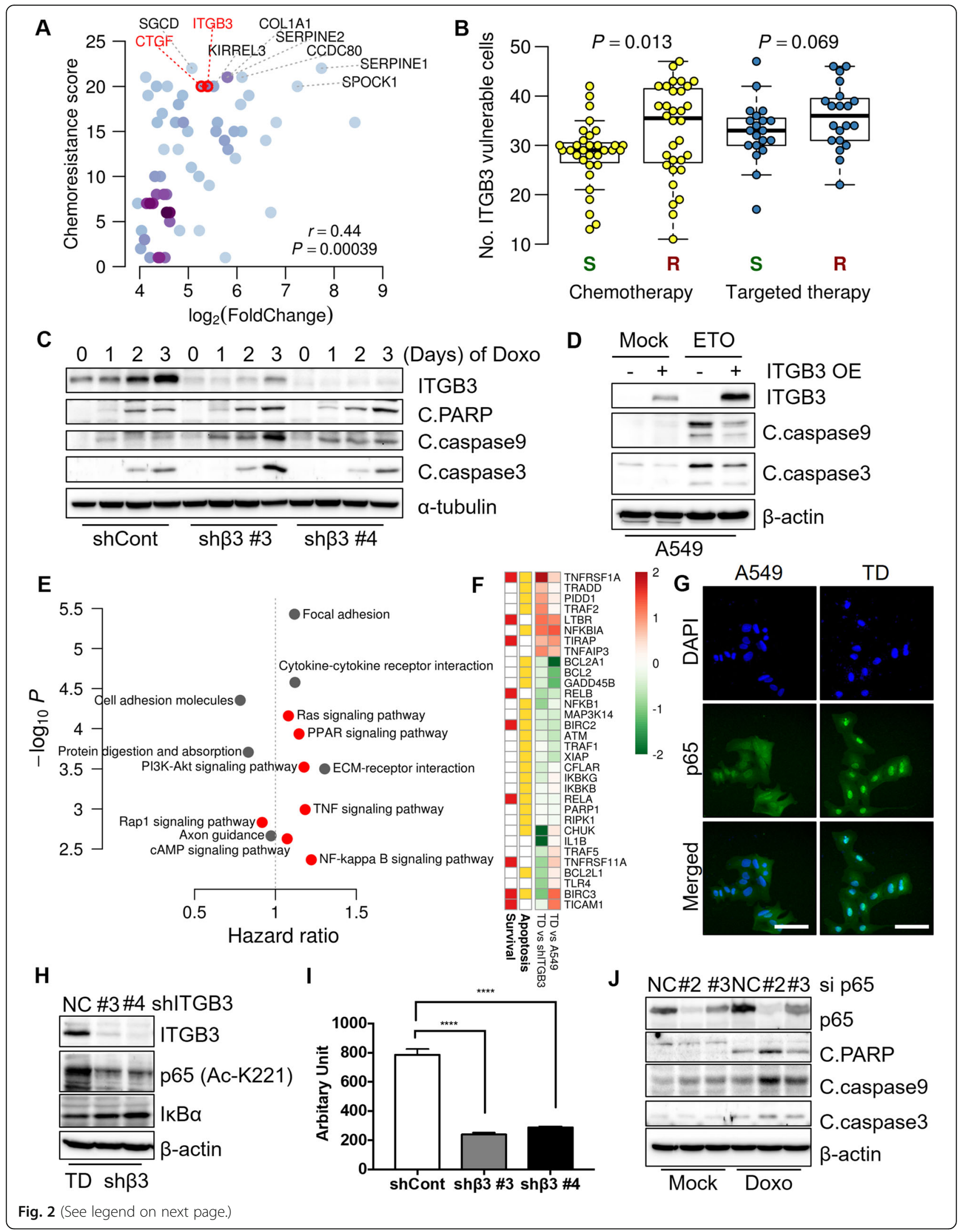




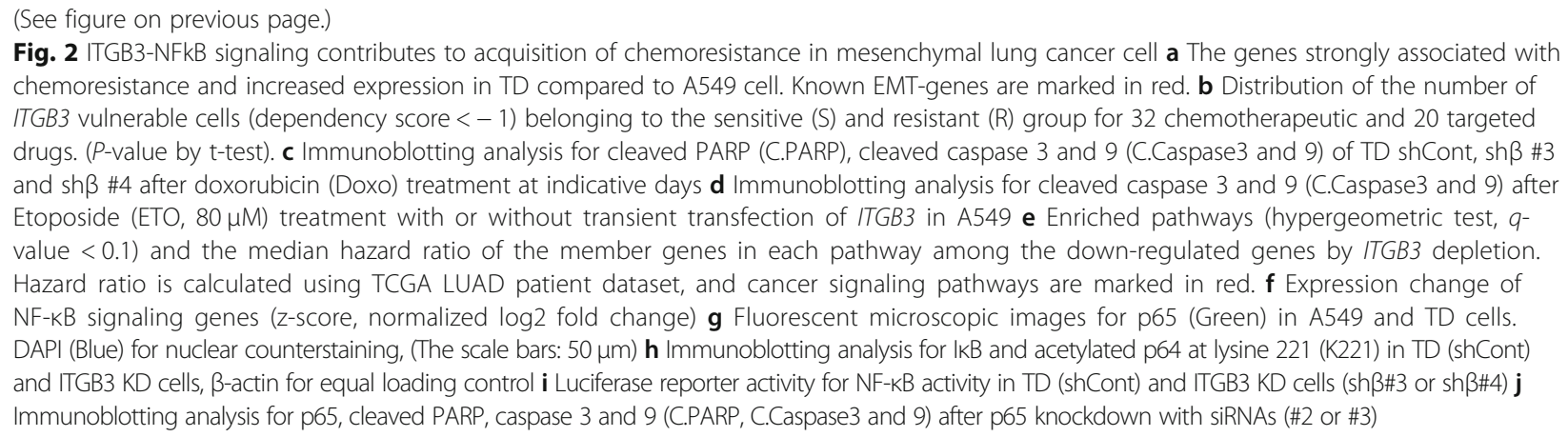

a major chemoresistance mechanism [1], we chose to investigate potential targets among the EMT signature genes, of which the vast majority were up-regulated by chemotherapeutics (Additional file 1: Figure S1C). Using the mesenchymal-type lung cancer cells (A549TD; hereafter, TD) from the A549 lung cancer cell line [4] showing a clear gene signature of 'hallmark of EMT' (Additional file 1: Figure S1E) and high resistance to etoposide treatment [5] (Additional file 1: Figure S1F), we showed the expression profiles of TD cells cluster together with those of doxorubicin-resistant cell lines, whereas the parental line A549 with the sensitive cells (Fig. 1b). As predicted, high resistance of TD cells to other conventional chemoradiotherapies such as etoposide (ETO), camptothecin (CPT) (Fig. 1c), ionizing radiation (IR) (Fig. 1d) and doxorubicin (Fig. 1e-f), all of which trigger apoptosis by inducing DNA damage.

For prediction of genes for chemoresistance, we defined the frequency of a gene in the up-regulated DEGs as its 'chemoresistance score'; this value was strongly correlated with the degree of overexpression in TD (Spearman correlation $=0.44, P<0.0004$, Fig. 2a). Two genes that satisfied all three of the criteria; i) genes frequently represented in the up DEGs of the 32 chemotherapeutic drugs; ii) genes overexpressed in TD cells; and iii) EMT-related genes were ITGB3 and CTGF (Fig. 2a). Of note, the role of CTGF in chemoresistance and its antagonism for chemosensitization has been determined [6]. In particular, we found that the $\mathrm{IC}_{50}$ of doxorubicin is positively correlated with the ITGB3 expression level (Additional file 1: Figure S2A). Analyzing a genome-scaling RNAi screening for 501 cancer cells, Project Achilles [7], 105 cells (21\%) showed significant dependency on ITGB3 (Additional file 1: Figure S2B), which was among the top $~ 10 \%$ vulnerable genes (Additional file 1: Figure S2C). Moreover, ITGB3-dependency was more significant in the resistant cells for most chemotherapeutic drugs (Fig. 2b and Additional file 1: Figure S2D). Such trend was most evident in eight chemotherapeutic drugs (Fisher's method $P<1.1 \times 10^{-5}$, Additional file 1: Figure S2E). Consistently, loss of ITGB3 in TD cells ( $\operatorname{sh} \beta \# 3$ and $\operatorname{sh} \beta \# 4$ ) (Additional file 1: Figure S2F) increased sensitivity to doxorubicin treatment (Fig. 2c and Additional file 1: Figure S2G-I). Similar results were obtained with CPT, IR, and ETO (Additional file 1: Figure S2J-L). Given that expression of ITGB3 was sufficient to restore the chemoresistance of $\operatorname{sh} \beta \# 3$ TD cells (Additional file 1: Figure S2M) and even increase the chemoresistance of A549, the parental cell line of TD (Fig. 2d), we conclude that ITGB3 expression is solely sufficient to induce chemoresistance.

Among the down-regulated pathways by ITGB3 depletion, NF- $\kappa \mathrm{B}$ was the signaling pathways most strongly associated with patients' survival (Fig. 2e). Given that depletion of ITGB3 down-regulated NF-kB-dependent survival factors (IL8, XIAP, PLAU, BIRC2/3, BCL2, or $B C L 2 L 1)$, and induced negative feedback regulators such as NFKBIA and TNFAIP3 (encoding I $\mathrm{I} B \alpha$ and A20 deubiquitinase, respectively), we hypothesized that inhibition of NF- $\mathrm{kB}$ signaling would be a key process required for cell sensitization (Fig. $2 \mathrm{f}$ and Additional file 1: Figure S3A). Consistently, highly chemoresistant TD cells exhibited higher levels of NF- $\mathrm{kB}$ activity than A549 cells, as determined by nuclear p65 localization (Fig. $2 \mathrm{~g}$ and Additional file 1: Figure S3B), the protein level of $\mathrm{I}_{\kappa} \mathrm{B} \alpha$ (Additional file 1: Figure S3C) and NF- $\mathrm{kB}$ reporter activity (Additional file 1: Figure S3D). Furthermore, loss of ITGB3 markedly attenuated NF- $\mathrm{B}$ activity, as determined by NF- $\mathrm{kB}$ reporter activity (Fig. 2h) and acetylation of p65 (which is critical for its DNA-binding affinity) (Fig. 2i) as well as the level of nuclear p65 (Additional file 1: Figure S3E-F). In adverse, ectopic expression of ITGB3 restored NF- $\kappa \mathrm{B}$ activity, as determined by acetylation of p65 and level of IאB (Additional file 1: Figure S3G). Together, these data indicate that ITGB3 expression is closely associated with NF- $\kappa B$ activity. According to this prediction that elevation of NF-kB activity by ITGB3 expression could be a primary cause of the elevated hazard ratio (Fig. 2e), we assessed the cytotoxicity of doxorubicin following abrogation of NF- $\mathrm{kB}$ activity. Depletion of p65 with siRNA, was sufficient to sensitize cells to doxorubicin treatment (Fig. 2j), suggesting that the increase in NF-kB 
A

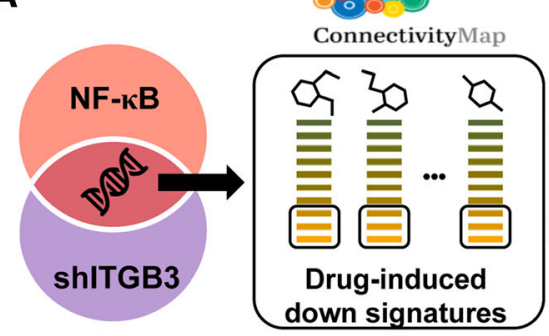

ITGB3 Signatures

\section{down signatures}

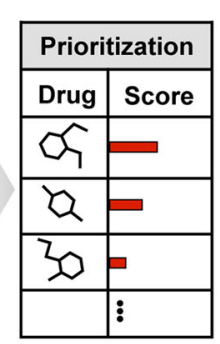

B

\begin{tabular}{lr}
\multicolumn{1}{c}{ shlTGB3 } & NF-кB \\
Naftidrofuryl & Phenothiazine \\
Enzastaurin & Danazol \\
Fluticasone & Atorvastatin \\
Fluorometholone & Methylprednisolone \\
&
\end{tabular}

C

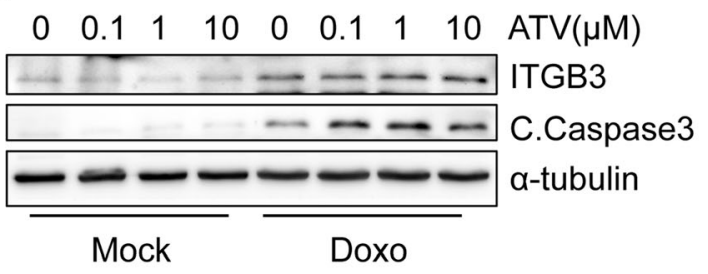

D

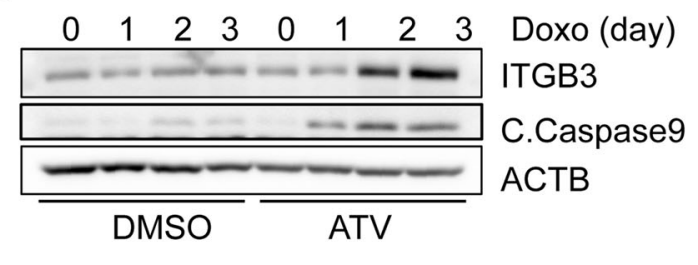

E
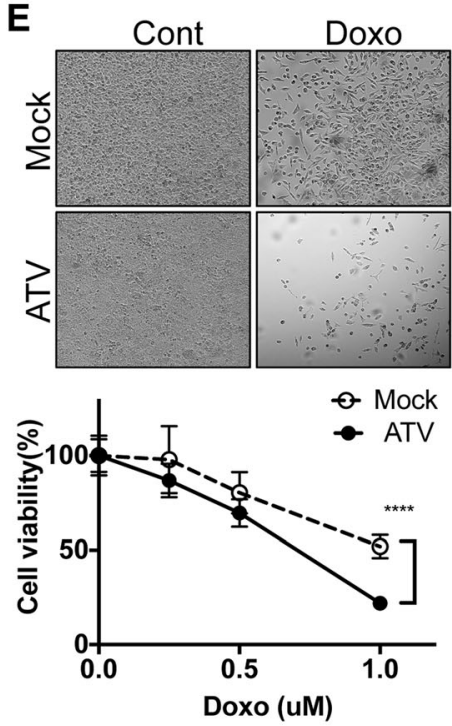

F
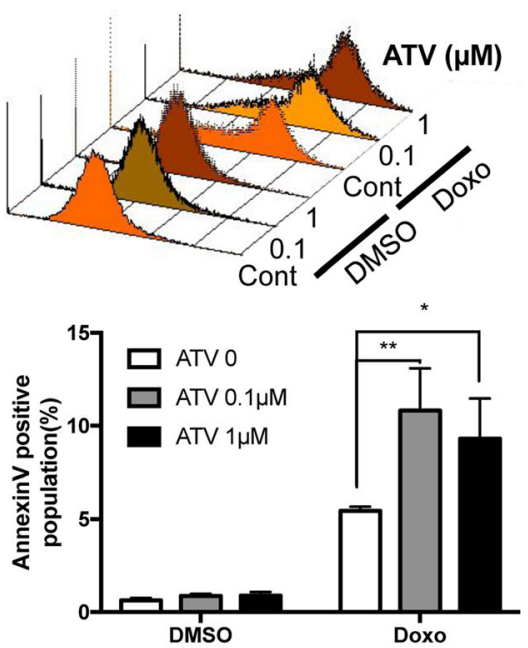

G

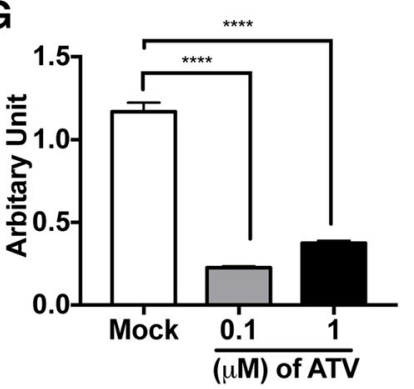

H

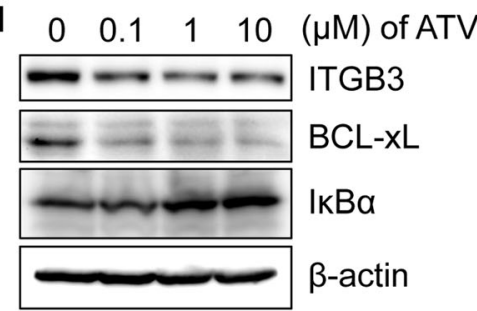

Fig. 3 Atrovastatin sensitizes chemotherapy through modulating NF-KB a CMap approach to identify chemosensitizer drugs using two different signatures: i) down-regulated genes by ITGB3 depletion, and ii) the intersection of i) and NF-KB pathway genes $\mathbf{b}$ the candidate drug list predicted by the two signatures. Atorvastatin was commonly predicted by both signatures. $\mathbf{c}$ and $\mathbf{d}$ Immunoblotting analysis for cleaved caspase 3 or (C.Caspase3 or C.Caspase9) at indicative dose $\mathbf{c}$ of atorvastatin (ATV) or Days (d, with $0.1 \mu \mathrm{M}$ of ATV) with doxorubicin (Doxo). a-tubulin or $\beta$-actin for equal loading control e Light microscopic images of TD cells with or without atorvastatin (ATV, $1 \mu \mathrm{M}$ ) after doxorubicin treatment (Doxo) (top), Graphical presentation of cell viability (bottom) f Flow cytometry for Annexin $V$ staining at $24 \mathrm{~h}$ after indicative dose of Doxorubicin (Doxo) with $0.1 \mu \mathrm{M}$ of ATV pretreatment (top), Graphical presentation of apoptotic cells (bottom) $\mathbf{g}$ Luciferase reporter activity for NF-KB activity in TD after indicative dose of atorvastatin (ATV) $\mathbf{h}$ Immunoblotting analysis for ITGB3, BCL-xL and IKB after indicative dose of atorvastatin (ATV) treatment in TD cells, $\beta$-actin for equal loading control 
activity mediated by ITGB3 expression is responsible for acquisition of chemoresistance.

To predict chemosensitizing drug candidates, we leveraged drug-induced transcriptome data from the Connectivity Map (CMap) and searched for drugs with expression signatures similar to those of ITGB3 depletion or NF- $\mathrm{kB}$ signaling inhibition (Fig. 3a). Among the candidate drugs, atorvastatin (ATV) was the only drug identified based on both ITGB3 and NF-kB gene signatures (Fig. $3 \mathrm{~b}$ and Additional file 1: Figure S4A-B). As predicted, pretreatment with ATV significantly sensitized TD cells to doxorubicin (Fig. 3c). It is noteworthy that, despite clear induction of ITGB3 by treatment of doxorubicin, combined treatment of ATV and doxorubicin increased the rate of cell death (Fig. 3d), decreased cell viability (Fig. 3e) and apoptotic cell death (Fig. 3f). The increase in chemosensitivity following ATV treatment occurred in parallel with a reduction in NF- $\mathrm{kB}$ reporter activity (Fig. 3g). As similar as induction of NFKBIA (encoding $I \kappa B \alpha$ ) and suppression of BCL2L1 (encoding BCL-xL) by ITGB3 depletion (Additional file 1: Figure S3A), which may account for the decrease in NF- $\mathrm{kB}$ activity as well as pro-survival activity, short-course ATV treatment increased expression of IкB $\alpha$ and decreased the level of BCL-xL (Fig. 3h), suggesting that attenuation of the NF- $\mathrm{kB}$-dependent pro-survival pathway by ATV leads to chemosensitization. Consistently, H460 cancer cells with mesenchymal gene expression (Additional file 1: Figure S5A) and high ITGB3 and IL6 expression (Additional file 1: Figure S5B), which was repressed by loss of ITGB3 (Additional file 1: Figure S5C and D) became more chemosensitive by ATV treatment (Additional file 1: Figure S5F), lowing NF- $\mathrm{kB}$ activity (Additional file 1: Figure S5E). In other hand, H358 cancer cells with epithelial gene expression (Additional file 1: Figure S5G) were likely to acquire chemoresistance (Additional file 1: Figure $\mathrm{S} 5 \mathrm{H}$ ) and increased NF- $\mathrm{kB}$ activation (Additional file 1: Figure S5I) by ITGB3 ectopic expression, which were weakened by ATV treatment (Additional file 1: Figure S5H and I). Conversely, depletion of ITGB3 promoted chemosensitivity in H358 (Additional file 1: Figure S5J).

Most targets responsible for acquired chemoresistance in cancers, identified during extensive molecular mechanistic studies, remain undrugged [8] due to poor druggability or possible side effects by direct inhibition. Thus, we took advantage of CMap approach based on a large-scale drug-induced transcriptome dataset and identified ATV, one of the world's best-selling drugs for hyperlipidemia, as a candidate drug for abrogating the pro-survival and chemoresistance effect of ITGB3; specifically, we showed that the transcriptional profile of ATV-treated cells was similar to that of ITGB3 knockdown. Consistently, ATV has a radiosensitizing effect on prostate cancer cells [9]. Although the inhibitory effect of STATINs on NF-kB is varied markedly [10], our predictive analysis identified ATV as a top-ranking candidate, strongly validating our data-driven approach.

\section{Conclusions}

By integrating pharmacogenomics and chemical genomic data, we successfully identified both a therapeutic target and a novel chemosensitizing drug to overcome resistance to multiple chemotherapeutic drugs. Our approach can be applied to a wide range of targets beyond those associated with EMT, paving an alternative path to drug discovery even for undruggable targets.

\section{Additional file}

Additional file 1: Supplementary Materials, Methods, and Figures

(Figure S1-S5). (PDF 5239 kb)

\section{Abbreviations}

ATV: Atorvastatin; AUC: Area under fitted curve; CCLE: Cancer Cell Line Encyclopedia; CMap: Connectivity Map; CPT: Camptothecin; CS: Chemoresistance score; CTGF: Connective Tissue Growth Factor; CTRP: Cancer Therapeutics Response Portal; DEG: Differentially expressed gene; EMT: Epithelial-mesenchymal transition; GDC: Genomics Data Common; GDSC: Genomics of Drug Sensitivity in Cancer; ITGB3: Integrin beta 3

\section{Acknowledgements}

We appreciate Seon-Young Kim and Dong-Uk Kim at Korea Research Institute of Bioscience and Biotechnology (KRIBB) for helpful discussions.

\section{Funding}

This work was supported by a grant from the National Research Foundation of Korea (NRF-2017M3C9A5028691 from HJC, NRF-2017M3C9A5028690 from WKK and NRF-2017R1A6A3A11030794 from HSL).

\section{Availability of data and materials}

The datasets used and/or analyzed during the current study are available from the corresponding author on reasonable request.

\section{Authors' contributions}

HJC and WK conceived the overall study design and led the experiments. SKH, HL, OSK, NYS, HJL, SK, JHK and MK conducted the experiments, data analysis, and critical discussion of the results. All authors contributed to manuscript writing and revising, endorsed and approved the final manuscript.

Ethics approval and consent to participate

Not applicable.

Consent for publication

Not applicable.

Competing interests

The authors declare that they have no competing interests.

\section{Publisher's Note}

Springer Nature remains neutral with regard to jurisdictional claims in published maps and institutional affiliations.

\section{Author details}

${ }^{1}$ College of Pharmacy, Seoul National University, Seoul 08826, Republic of Korea. 'Department of Life Sciences, Ewha Womans University, Seoul 03760, Republic of Korea. ${ }^{3}$ College of Natural Sciences, Department of Life Sciences, Sogang University, Seoul 04107, Republic of Korea. ${ }^{4}$ Personalized Genomic Medicine Research Center, Korea Research Institute of Bioscience and Biotechnology, Daejeon 34141, Republic of Korea. 
Received: 11 July 2018 Accepted: 30 November 2018

Published online: 18 December 2018

\section{References}

1. Fischer KR, Durrans A, Lee S, Sheng J, Li F, Wong ST, Choi H, El Rayes T, Ryu $\mathrm{S}$, Troeger J, et al. Epithelial-to-mesenchymal transition is not required for lung metastasis but contributes to chemoresistance. Nature. 2015;527:472-6.

2. De Sousa E, Melo F, Wang X, Jansen M, Fessler E, Trinh A, de Rooij LPMH, de Jong JH, de Boer OJ, van Leersum R, Bijlsma MF, et al. Poor-prognosis colon cancer is defined by a molecularly distinct subtype and develops from serrated precursor lesions. Nat Med. 2013;19:614-8.

3. Sistigu A, Yamazaki T, Vacchelli E, Chaba K, Enot DP, Adam J, Vitale I, Goubar A, Baracco EE, Remedios C, et al. Cancer cell-autonomous contribution of type I interferon signaling to the efficacy of chemotherapy. Nat Med. 2014; 20:1301-9.

4. Hong SK, Park JR, Kwon OS, Kim KT, Bae GY, Cha HJ. Induction of integrin beta3 by sustained ERK activity promotes the invasiveness of TGFbetainduced mesenchymal tumor cells. Cancer Lett. 2016;376:339-46.

5. Kwon O-S, Hong S-K, Kwon S-J, Go Y-H, Oh E, Cha H-J. BCL2 induced by LAMTOR3/MAPK is a druggable target of chemoradioresistance in mesenchymal lung cancer. Cancer Lett. 2017;403:48-58.

6. Neesse A, Frese KK, Bapiro TE, Nakagawa T, Sternlicht MD, Seeley TW, Pilarsky C, Jodrell DI, Spong SM, Tuveson DA. CTGF antagonism with mAb FG-3019 enhances chemotherapy response without increasing drug delivery in murine ductal pancreas cancer. Proc Natl Acad Sci U S A. 2013; 110:12325-30

7. Tsherniak A, Vazquez F, Montgomery PG, Weir BA, Kryukov G, Cowley GS, Gill S, Harrington WF, Pantel S, Krill-Burger JM, et al. Defining a cancer dependency map. Cell. 2017;170:564-76 e516.

8. Dang CV, Reddy EP, Shokat KM, Soucek L. Drugging the 'undruggable' cancer targets. Nat Rev Cancer. 2017;17:502-8.

9. He M, Hu J, Xia Y. Large scale expressed sequence tag (EST) analysis of Metarhizium acridum infecting Locusta migratoria reveals multiple strategies for fungal adaptation to the host cuticle. Curr Genet. 2012;58:265-79.

10. Hilgendorff A, Muth H, Parviz B, Staubitz A, Haberbosch W, Tillmanns H, Holschermann $\mathrm{H}$. Statins differ in their ability to block NF-kappaB activation in human blood monocytes. Int J Clin Pharmacol Ther. 2003;41:397-401.

Ready to submit your research? Choose BMC and benefit from:

- fast, convenient online submission

- thorough peer review by experienced researchers in your field

- rapid publication on acceptance

- support for research data, including large and complex data types

- gold Open Access which fosters wider collaboration and increased citations

- maximum visibility for your research: over $100 \mathrm{M}$ website views per year

At $\mathrm{BMC}$, research is always in progress.

Learn more biomedcentral.com/submissions 